\title{
A New High-Efficiency Experimental Design for Optimizing Various Flow Velocities Testing in Extremely Aggressive Formation Water
}

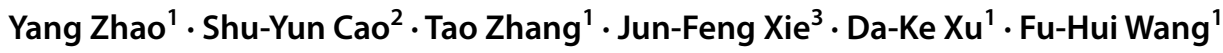

Received: 23 March 2019 / Revised: 12 April 2019 / Published online: 20 June 2019

(c) The Chinese Society for Metals (CSM) and Springer-Verlag GmbH Germany, part of Springer Nature 2019

\begin{abstract}
Compared to the traditional one-by-one method, a new high-efficiency method is used to characterize large numbers of regulations varying samples. Accordingly, bump-shaped electrodes are designed using the computational fluid dynamics model, and the effect of the height and placement of these electrodes is discussed. The experimental feasibility is certified by weight loss measurement. Results indicate that flow velocities of different bump-shaped electrode surfaces are significant differences. Thus, each surface can be analyzed independently; the thickness loss of each electrode surface is consistent with that using one-by-one method, which can effectively improve the experimental efficiency 12 times.
\end{abstract}

Keywords Bump-shaped electrodes · High efficiency · Computational fluid dynamics (CFD) · Weight loss

\section{Introduction}

The aggressive environment is a big challenge to the gas exploitation in the west of China, which is characteristic of extra high temperature, high salinity and high $\mathrm{CO}_{2}$ partial pressure [1]. In the presence of salinity brine, $\mathrm{CO}_{2}$ at high temperature and elevated pressure can form highly aggressive medium with low $\mathrm{pH}$, which would increase the risk of tube leakage and catastrophic accident, leading to huge economic loss [2]. Accordingly, it is essential to evaluate $\mathrm{CO}_{2}$ corrosion risk of well tubes in the exploitation process.

Available online at http://link.springer.com/journal/40195

Yang Zhao and Shu-Yun Cao have equally contributed to this work.

Tao Zhang

zhangtao@mail.neu.edu.cn

1 Shenyang National Laboratory for Materials Science, Northeastern University, 3-11 Wenhua Road, Shenyang 110819, China

2 State Key Laboratory of Separation Membranes and Membrane Processes, School of Material Science and Engineering, Tianjin Polytechnic University, Tianjin 300387, China

3 Petrochina Tarim Oilfield Company, Shihua Road, Korla 841000, China
In fact, $\mathrm{CO}_{2}$ corrosion of well tubes is a quite complex process, which involves multiple chemical and electrochemical reactions. Its corrosion mechanism and corrosion rate can be affected by many factors, such as $\mathrm{CO}_{2}$ partial pressure, temperature, $\mathrm{pH}$ and flow velocity [3-6]. Among the above factors, the flow velocity is the most critical parameter, especially for determining the suitability of the tubes [7, 8]. Plenty of failure cases of stainless steel (SS) caused by the flow velocity had been reported on well-established facts [9-13]. However, the previous researches mainly focused on low $\mathrm{CO}_{2}$ partial pressure $(<2 \mathrm{MPa})$, low temperature $\left(<100{ }^{\circ} \mathrm{C}\right)$ and low salinity environments $\left(\mathrm{Cl}^{-}\right.$concentration $<10^{4} \mathrm{mg} / \mathrm{L}$ ) [14-17]. The main reasons of the aforementioned may be due to the big challenge of the equipment and the testing methods.

To narrow the research gap, in recent years, the technology of rotating cage, which normally provides controlled mass transfer in flow for electrode kinetic studies, has developed as a compact, hydrodynamic experimental method [18]. Moreover, the maximum flow velocity is limited owing to the reliability of the equipment. Accordingly, the flow loop is designed to approach understanding the corrosion process under flow conditions [19]. However, compared to that of rotating cage, the flow loop is less convenient, efficient and more difficult to conduct. Moreover, a highefficiency experimental method and design resources from the materials genome initiative (MGI) have been proposed 
to combine the merit of both the rotating cage and the flow loop technology.

The MGI can compute, produce and construct fundamental databases. It combines high-efficiency theoretical calculations with the inclusion of the functions for prediction, screening and optimization of materials by data mining from existing database [20]. The objective is to accelerate the design of new materials in an unprecedented scale and rate with low cost [21]. The design of using the high-efficiency experimental method is one of the modules of the MGI, which can be regarded as a preparation technique. This method and design have been applied to many fields and supported by many open databases [22-24], such as diffusion multiple approaches [25], thin-film deposition [26], the development of cladding materials [27] and the measurement of mechanical properties of samples [28].

Therefore, in the present study, the selected HP-13Cr SS, having a higher pitting corrosion resistance and excellent mechanical properties, is used in the down-hole strings. A new high-efficiency experimental method is established, which shows a relatively high efficiency, by combining the rotating cage and flow loop to conduct the various flow velocities testing in the extremely aggressive environment. Furthermore, the effect of the height, the placement and the reliability of the bump-shaped electrodes are discussed by using the computational fluid dynamics (CFD) simulation. In addition, a highly accurate correlation between the theoretical simulation and experimental results is supported by the founding of the weight loss measurement.

\section{Experimental}

\subsection{Material and Solution}

HP-13Cr SS, with its composition (wt\%) of $0.003 \mathrm{C}, 0.15$ $\mathrm{Si}, 0.51 \mathrm{Mn}, 12.77 \mathrm{Cr}, 2.19 \mathrm{Mo}, 0.002 \mathrm{~S}, 0.02 \mathrm{P}, 0.047 \mathrm{Cu}$, $5.36 \mathrm{Ni}, 0.014 \mathrm{~V}, 0.037 \mathrm{Al}$ and $\mathrm{Fe}$ balance, is used for the following experimental study.

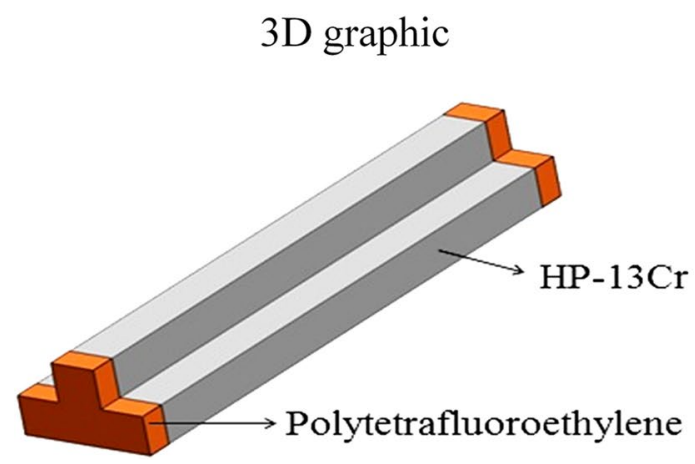

Fig. 1 Schematic of the bump-shaped electrode
The testing electrolyte, simulating the formation water, was prepared using analytical grade reagents and deionized water $(18 \mathrm{M} \Omega / \mathrm{cm}$ in resistivity). Its chemical composition $(\mathrm{mg} / \mathrm{L})$ is $189 \mathrm{HCO}_{3}{ }^{-}, 128000 \mathrm{Cl}^{-}, 430 \mathrm{SO}_{4}{ }^{2-}, 8310 \mathrm{Ca}^{2+}$, $561 \mathrm{Mg}^{2+}, 6620 \mathrm{~K}^{+}$and $76500 \mathrm{Na}^{+}$.

\subsection{Weight Loss Measurement}

The immersion experiments of the HP- $13 \mathrm{Cr}$ SS in the prepared formation water were carried out for 30 days in a hightemperature and high-pressure dynamic stainless autoclave with a rotating cage installed. The rotating cage is designed to imitate actual flow conditions, with rotation speed up to $810 \mathrm{rpm}$ (corresponding to a calculated flow speed of $3 \mathrm{~m} / \mathrm{s}$ over the specimen surface).

In general, the first critical step of application of the highefficiency experimental design to optimize multiple flow velocities is the design of the electrodes used in the highly aggressive formation water. The schematic diagram of the bump-shaped electrode is shown in Fig. 1 and marked the surface of the bump-shaped electrode from No. 1 to No. 6 clockwise direction. In particular, No. 6 surface was toward the center axis when the bump-shaped electrode was fixed at the rotate cage. Both ends of the bump-shaped electrodes are covered with polytetrafluoroethylene in order to prevent corrosion. Moreover, the surface covered with polytetrafluoroethylene can be used as the reference surface to evaluate the thickness loss of each electrode surface encountering varied flow velocities.

After the immersion experiments, the specimens covered with polytetrafluoroethylene were taken out of the formation water and rinsed with deionized water and anhydrous ethanol, respectively. Three aforementioned parallel specimens were descaled in the solution consisting of nitric acid $\left(100 \mathrm{~mL}, \rho\right.$ is $\left.1.42 \mathrm{~g} \mathrm{~mL}^{-1}\right)$, hydrofluoric acid $(20 \mathrm{~mL}, \rho$ is $\left.1.15 \mathrm{~g} \mathrm{~mL}^{-1}\right)$ and deionized water $(880 \mathrm{~mL})$ at room temperature according to the ASTM standard G1-03 [29] to remove the sales film and polytetrafluoroethylene from the electrode surface.

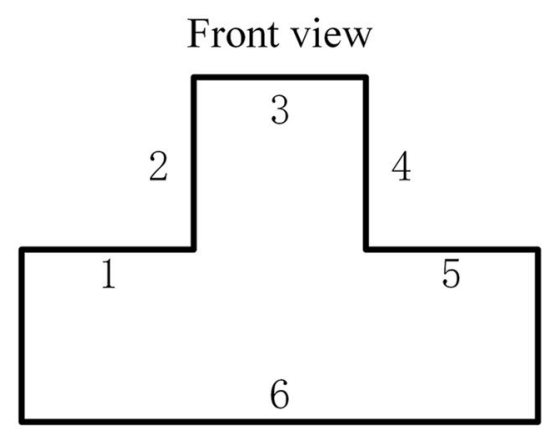


Subsequently, the thickness loss of each surface of the bump-shaped electrode was obtained from the super deep scene 3D microscope (VHX-5000, Japan). The super deep scene 3D microscope has the function of the macroscopic mosaic. The outline of the surfaces both covered and uncovered by polytetrafluoroethylene could be simultaneously achieved and overlapped. As a result, the corrosion rate $\left(V_{\mathrm{c}}\right)$ can be calculated using the following equation:

$V_{\mathrm{c}}=\Delta H / t$,

where $\Delta H$ is the thickness loss of each surface of the bumpshaped electrode $(\mathrm{mm}), t$ is the immersion time. To ensure reproducibility, each measurement was repeated at least three times. Average corrosion rates with error bars were calculated based on three parallel specimens for each test.

\subsection{Fluent Model Simulation}

Numerical simulation of the flow field was conducted on the commercial software commercial CFD package, FLUENT, which is based on the finite volume approach. The SST $k-\omega$ turbulent model is used for approaching the governing equations. The surface of the specimen is defined as a non-slip solid wall with a rotation speed of $75 \mathrm{rad} / \mathrm{s}$. The iterative calculations of the semi-implicit method for pressure-linked equations (SIMPLE) algorithm were used to solve the momentum equation ( $N-S$ equations), which can modify the pressure term in the discretized $N-S$ equations so as to renew the velocity field and the pressure field simultaneously. The iterative calculations of the primitive variables such as flow velocity are terminated when the residual norm criteria, $10^{-6}$, was reached.

\section{Results and Discussion}

\subsection{Effect of the Height of the Bump-Shaped Electrodes Under the Flow Condition}

The velocity distribution of the bump-shaped electrodes with various heights is shown in Fig. 2, and the average flow velocity for each surface is given in Table 1. It can be seen that, regardless of the height of the bump-shaped electrode, the vortex flow forms and varies obviously when the formation water flows through each electrode surface of the bump-shaped electrode. As a consequence, the average flow rate for each surface is different with the color changes; thus, each surface can be analyzed independently. Furthermore, the diversity of the average flow rate for each surface becomes much more pronounced with an increase in the height of the bump-shaped electrodes from 1 (Fig. 2a) to $3 \mathrm{~mm}$ (Fig. 2c). Given that the flow velocities of surfaces of different bump-shaped electrodes are significant differences, it can be indicated that the bump-shaped electrodes are suitable to optimize the flow velocity testing, and the experimental efficiency can be improved. Furthermore, when the height of the bump-shaped is high enough $(3 \mathrm{~mm})$, each surface can be regarded most readily as an independent individual to be analyzed because of the biggest difference of the various velocities for the electrode surfaces. Considering

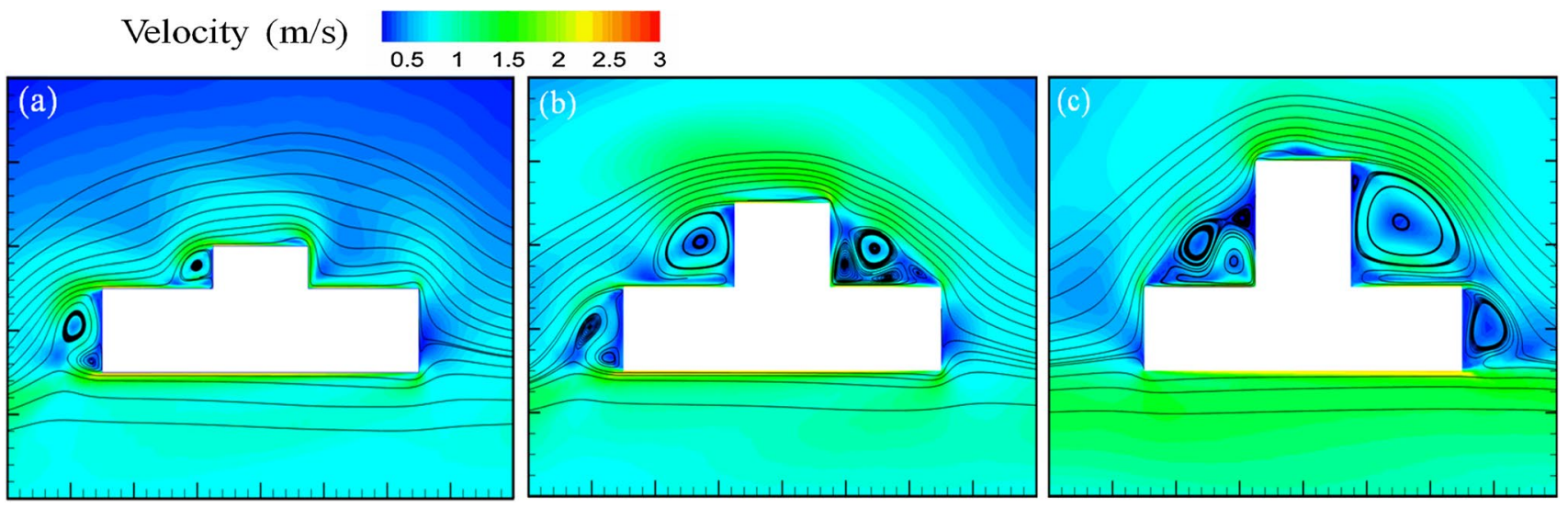

Fig. 2 Velocity distribution of the bump-shaped electrodes with various heights: a $1 \mathrm{~mm}, \mathbf{b} 2 \mathrm{~mm}, \mathbf{c} 3 \mathrm{~mm}$

Table 1 Average flow velocity for each surface of the bumpshaped electrode

\begin{tabular}{lllllll}
\hline Height $(\mathrm{mm})$ & No. 1 (m/s) & No. 2(m/s) & No. 3 (m/s) & No. 4 (m/s) & No. 5 (m/s) & No. 6 (m/s) \\
\hline 1 & 1.389 & 2.558 & 1.414 & 3.095 & 1.856 & 3.068 \\
2 & 1.404 & 2.865 & 1.454 & 3.105 & 2.165 & 3.054 \\
3 & 1.375 & 2.751 & 1.662 & 3.521 & 2.054 & 3.012 \\
\hline
\end{tabular}


the size of the rotating cage and experimental operability, a bump-shaped electrode with a height of $3 \mathrm{~mm}$ is preferable to be used in the experiment.

\subsection{Effect of the Placement of the Bump-Shaped Electrode on the Flow Condition}

The high-efficiency method is implemented by combing the rotate cage and the bump-shaped electrodes. When the rotate cage is settled, the arrangement of the bump-shaped electrodes on the rotate cage is a critical parameter to ensure the uniformity of the velocity flowing through each electrode surface. Therefore, the velocity distribution of the bumpshaped electrodes is designed to be arranged symmetrically and variedly, using the CFD simulation, which is shown in Fig. 3. Figure 3 clearly shows that the velocity field around the eight bump-shaped electrodes arranged on the rotating cage is uniform and homogeneous (Fig. 3a), because of the similar color distribution around these electrodes. However, the velocity field of the four bump-shaped electrodes and four flat electrodes (Fig. 3b) is not as consistent as that of the eight bump-shaped electrodes. Consequently, the inconsistent velocity field would bring some difficulties to calculate the average flow rate for each surface of the bump-shaped electrodes. Therefore, in the high-efficiency experiment, the bump-shaped electrodes should be uniformly arranged on the rotating cage so as to guarantee the accuracy of the highefficiency data.
The experimental efficiency is associated with the homogeneity of the flow condition around the bump-shaped electrodes. If the velocity distribution is uniform and each electrode can work independently, the experimental efficiency can be enhanced. In order to improve the experimental efficiency, the selection of the distance between the bumpshaped electrode and the center axis for each layer is also important, which affects the corresponding flow velocity [30]. It should be noted that the structure of the rotating cage can also affect the homogeneity of the flow condition around the bump-shaped electrodes [31], especially when the rotating cage is a single layer. Trying to not affecting the homogeneity of the flow condition around the bump-shaped electrodes and considering the dimensions and structure of the autoclave, the rotating cage is designed to a double layer, and the bump-shaped electrodes are arranged diversely but evenly for each rotating cage layer with the same axis. Figure 4 shows side views of the velocity distribution of the bump-shaped electrodes arranged at the double-layer rotate cage, obtained by the CFD simulation. It can be observed that using either the eight bump-shaped electrodes (Fig. 4a), or the four bump-shaped electrodes and four flat electrodes (Fig. 4b), the flow condition for each layer is unaffected by each other. Moreover, velocity distribution of the eight bump-shaped electrodes is also more uniform than that of the four bump-shaped electrodes and four flat electrodes. Therefore, it can be concluded that when using multilayer structure of the rotating cage (in this case, double-layer one), and adjusting the distance between the bump-shaped

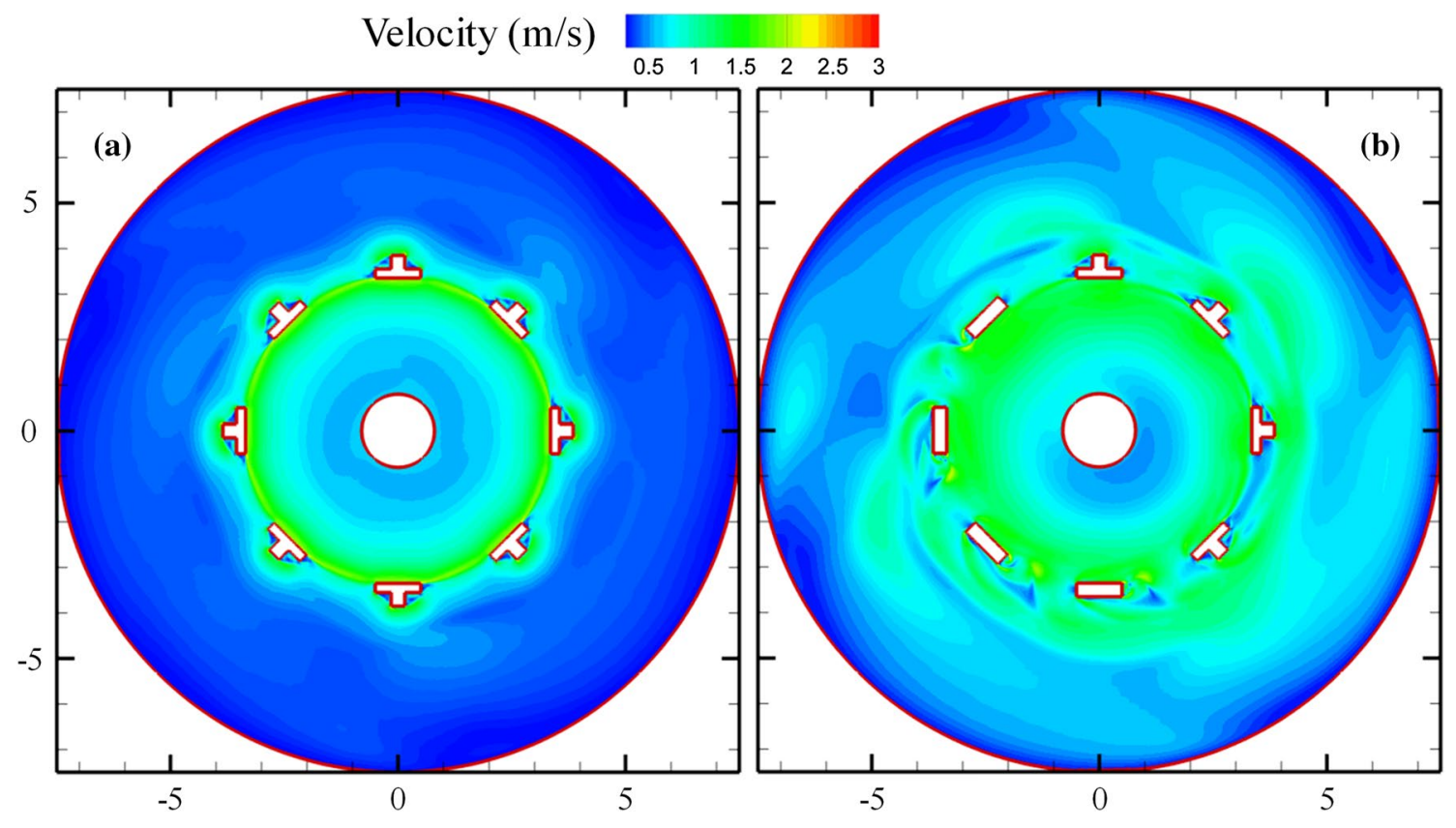

Fig. 3 Top views of the velocity distribution of the bump-shaped electrodes arranged symmetrically and variedly at the single rotate cage layer: a eight bump-shaped electrodes, $\mathbf{b}$ four bump-shaped electrodes and four flat electrodes 


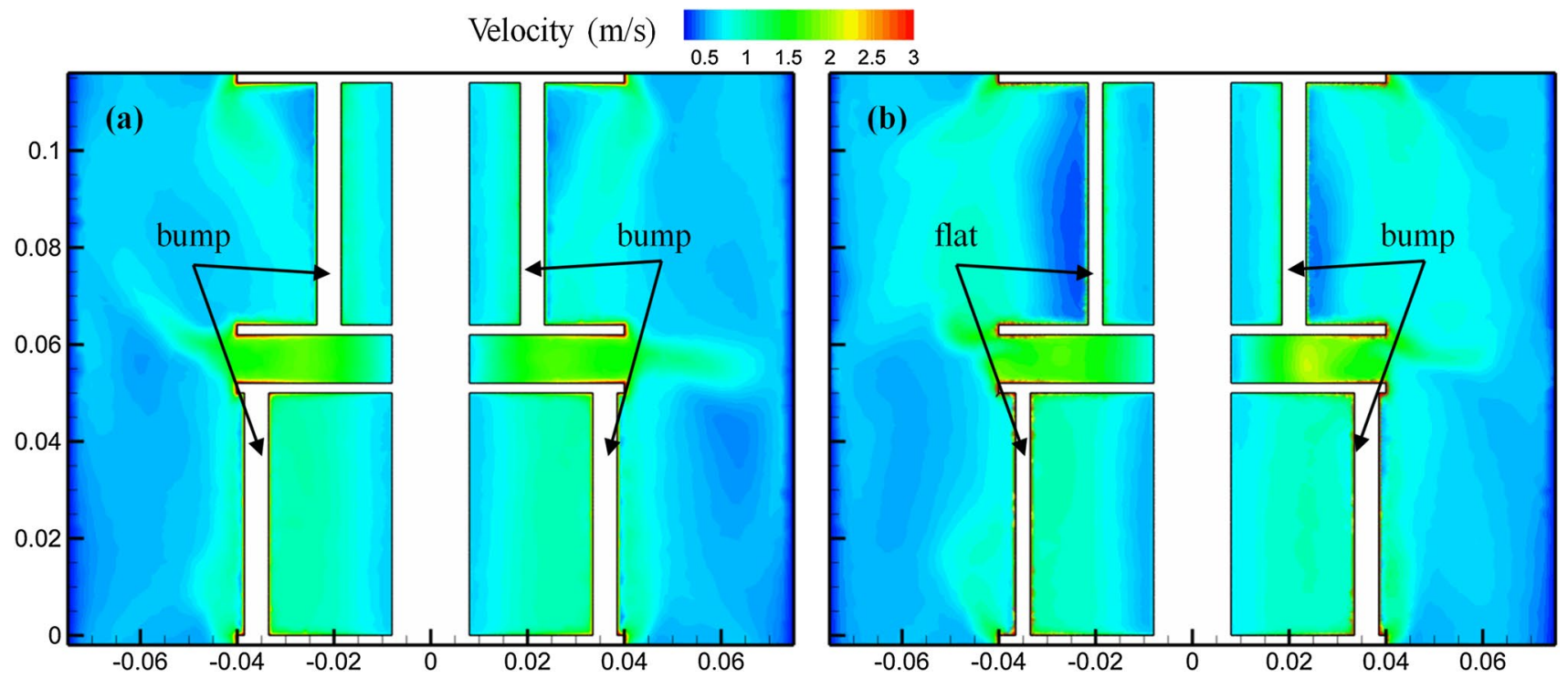

Fig. 4 Side views of the velocity distributions of the bump-shaped electrodes arranged symmetrically and variedly at the double-layer rotate cage: a eight bump-shaped electrodes, b four bump-shaped electrodes and four flat electrodes

electrodes and the center axis for each layer simultaneously, the design of this high-efficiency experiment is feasible, and the homogeneity of the flow condition around each electrode surface cannot be affected, leading to the enhanced efficiency of the high-efficiency experiment.

\subsection{Reliability of the Bump-Shaped Electrodes}

Besides certifying the feasibility of the bump-shaped electrodes with the CFD model, actually, the reliability of the electrodes is also important, which concerns their future application and needs to be further confirmed in practice. In this experiment, the traditional one-by-one method by weight loss tests is used for comparison. The super deep scene 3D microscope is generally used to examine the thickness loss of each surface of the bump-shaped electrode. The mosaicked macroscopic morphologies after removal of the corrosion scales are shown in Fig. 5. There is a significant difference in the thickness loss of each surface of the bumpshaped electrode. These surfaces are marked from number 1 to 6 (abbreviated Nos. 1 to 6) in clockwise order with an average thickness loss of each electrode surface 0.03 , $0.07,0.04,0.09,0.05$ and $0.08 \mathrm{~mm}$, respectively. Then, the thickness loss of each electrode surface is converted to $V_{\mathrm{c}}$ using Eq. (1). Figure 6 shows $V_{\mathrm{c}}$ as a function of different velocities obtained by both the traditional one-by-one method and this high-efficiency method. It can be seen that the $V_{\mathrm{c}}$ values obtained by these two different methods are nearly coincident. Therefore, as mentioned above, it can be concluded that it is reliable to conduct this high-efficiency experiments by combining using the bump-shaped electrodes and the rotating cage. Compared to that one-byone method, for the bump-shaped electrodes having six surfaces, the experimental efficiency can improve six times; for the up and down double layers of the rotating cage, the experimental efficiency can improve two times. Thus, the total experimental efficiency can improve $12(6 \times 2)$ times. Furthermore, based on the above discussion, it is possible to design much more complicated electrodes (not limited to the bump-shaped electrodes) and extra layers (more than double) of the rotating cage to further increase the experimental efficiency under extremely aggressive environment [32]. In addition, it is believable that the goal of the project of "high-efficiency experimental method and design" of the MGI would be realized in near future [33].

\section{Conclusions}

A new high-efficiency experiment is designed by combing selection of the bump-shaped electrodes and the doublelayer rotate cage to optimize the flow velocity testing in the extremely aggressive oilfield formation water. The above findings, based on discussing the effect of the height, the placement and the reliability of the bump-shaped electrodes, allow the following conclusions:

1. The bump-shaped electrode is designed to optimize the flow velocity testing. When the height of this kind of electrode is high enough, there is a significant differ- 

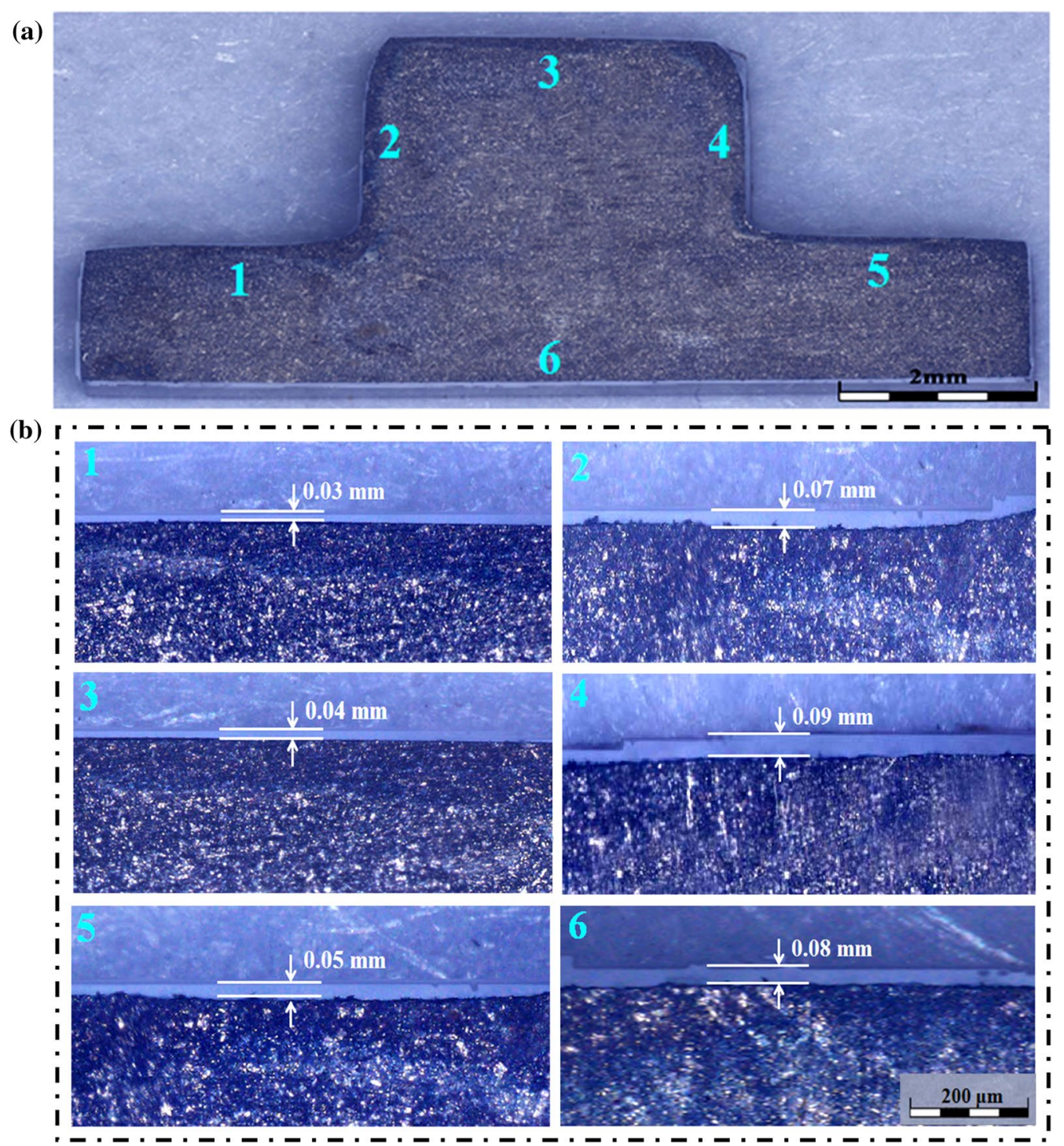

Fig. 5 Macroscopic morphologies of the bump-shaped HP-13Cr SS electrodes after removal of the corrosion scales in the extremely aggressive oilfield formation water: a global drawing, b partial enlarged drawing

ence of flow velocity for different bump-shaped electrode surfaces; each electrode surface can be analyzed independently.

2. A double-layer rotate cage is designed to ensure the homogeneity of the flow condition around the bumpshaped electrodes. The electrodes are evenly arranged with varied distances from the center axis for each rotate cage layer, and the flow field around these electrodes is unaffected by each other. The velocity distribution of the eight bump-shaped electrodes is more uniform than that of the four bump-shaped electrodes and four flat electrodes.

3. By comparison with weight loss tests using the one-byone method, the thickness loss is consistent with that using the traditional one-by-one method. However, the experimental efficiency is largely improved by using this designed high-efficiency experiment. 


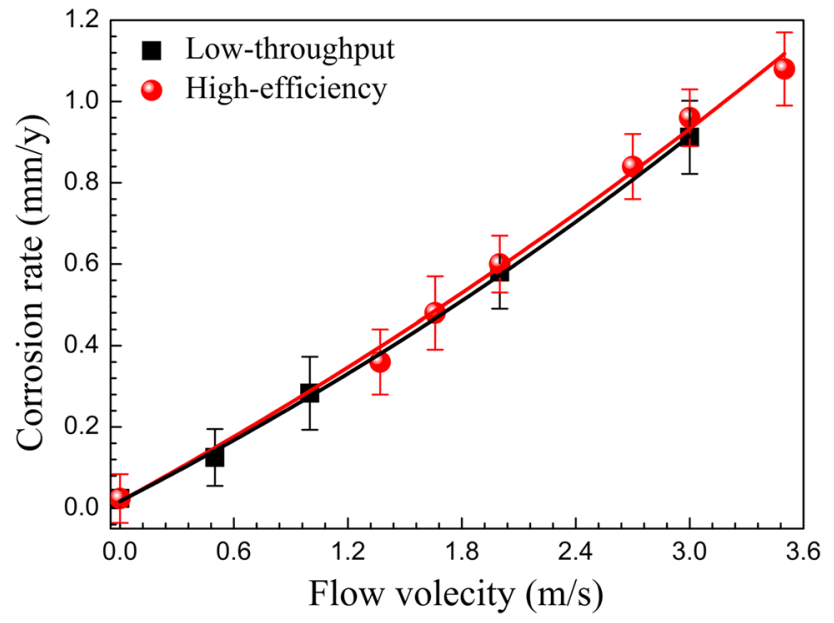

Fig. 6 Corrosion rates of the HP-13Cr SS electrodes at different velocities measured by the high-efficiency and traditional one-by-one methods

Acknowledgements The authors are grateful for the financial support of the National Key Research and Development Program of China (No. 2017YFB0702203), the National Natural Science Foundation of China (No. U1460202), the National program for the Young Top-notch Professionals and the Fundamental Research Funds for the Central Universities (No. N170205002).

\section{References}

[1] X.D. Lan, X.X. Lü, Y.M. Zhu, H.F. Yu, J. Nat. Gas. Sci. Eng. 22, 633 (2015)

[2] D. Sandana, M. Hadden, J. Race, E. Charles, J. Pipeline Eng. 11, $229(2012)$

[3] Y. Liu, L.N. Xu, M.X. Lu, Y. Meng, J.Y. Zhu, L. Zhang, Appl. Surf. Sci. 314, 768 (2014)

[4] A. Pfennig, A. Kranzmann, Corros. Sci. 65, 441 (2012)

[5] S.L. Lu, W. Liu, S.A. Zhang, X.L. Qi, X.G. Li, X.M. Wang, Acta Metall. Sin. (Engl. Lett.) 30, 1055 (2017)

[6] C.L. Wu, S. Zhang, C.H. Zhang, H. Zhang, S.Y. Dong, J. Alloys Compd. 698, 761 (2017)

[7] Q. Li, H.T. Hu, Y.F. Cheng, J. Petrol. Sci. Eng. 147, 408 (2016)
[8] W. Li, B.F.M. Pots, B. Brown, K.E. Kee, S. Nesic, Corros. Sci. 110, 35 (2016)

[9] S. Hassani, T.N. Vu, N.R. Rosli, S.N. Esmaeely, Y.S. Choi, D. Young, S. Nesic, J. Greenh. Gas Control 23, 30 (2014)

[10] A.Q. Liu, C. Bian, Z.M. Wang, X. Han, J. Zhang, Corros. Sci. 134, 149 (2018)

[11] Y. Long, G. Wu, A.Q. Fu, J.F. Xie, M.F. Zhao, Z.Q. Bai, J.H. Luo, Y.R. Feng, Eng. Fail. Anal. 93, 330 (2018)

[12] L. Fan, F.J. Tang, S.T. Reis, G. Chen, M.L. Koenigstein, Acta Metall. Sin. (Engl. Lett.) 30, 390 (2017)

[13] S. Zhang, C.L. Wu, C.H. Zhang, M. Guan, J.Z. Tan, Opt. Laser Technol. 84, 23 (2016)

[14] W. Sun, S. Nesic, R.C. Woollam, Corros. Sci. 51, 1273 (2009)

[15] F. Rouillard, T. Furukawa, Corros. Sci. 105, 120 (2016)

[16] B. Belkessa, D. Miroud, N. Ouali, B. Cheniti, Acta Metall. Sin. (Engl. Lett.) 29, 674 (2016)

[17] H. Zhang, C.H. Zhang, Q. Wang, C.L. Wu, S. Zhang, J. Chen, A.O. Abdullah, Opt. Laser Technol. 101, 363 (2018)

[18] F.C. Walsh, G. Kear, A.H. Nahlé, J.A. Wharton, L.F. Arenas, Corros. Sci. 123, 1 (2017)

[19] G.A. Zhang, L. Zeng, H.L. Huang, X.P. Guo, Corros. Sci. 77, 334 (2013)

[20] X.Y. Yang, Z.G. Wang, X.S. Zhao, J.L. Song, M.M. Zhang, H.D. Liu, Comput. Mater. Sci. 146, 319 (2018)

[21] J.C. Zhao, Adv. Eng. Mater. 3, 143 (2001)

[22] Y. Lederer, C. Toher, K.S. Vecchio, S. Curtarolo, Acta Mater. 159, 364 (2018)

[23] Y.X. Zhuang, W.J. Liu, P.F. Xing, F. Wang, J.C. He, Acta Metall. Sin. (Engl. Lett.) 25, 124 (2012)

[24] O.N. Senkov, J.D. Miller, D.B. Miracle, C. Woodward, Nat. Commun. 6, 1 (2015)

[25] J.C. Zhao, Annu. Rev. Mater. Res. 35, 51 (2005)

[26] Z.H. Barber, M.G. Blamire, Mater. Sci. Technol. 24, 757 (2008)

[27] Z. Liu, Y.F. Li, D.W. Shi, Y.L. Guo, M. Li, X.B. Zhou, Q. Huang, S.Y. Du, Scr. Mater. 141, 99 (2017)

[28] S.V. Kalinin, B.G. Sumpter, R.K. Archibald, Nat. Mater. 14, 973 (2015)

[29] ASTM Standard G1-03, (West Conshohocken, PA: ASTM International, 2003)

[30] G. Kear, B.D. Barker, K.R. Stokes, F.C. Walsh, Corros. Sci. 47, 1694 (2005)

[31] G.A. Zhang, Y. Zeng, X.P. Guo, F. Jiang, D.Y. Shi, Z.Y. Chen, Corros. Sci. 65, 37 (2012)

[32] S. Tsuboi, T. Tanaka, K.Y. Tamura, H. Kitamoto, J. Microbiol. Methods 146, 22 (2018)

[33] Z. Liu, Y.F. Li, D.W. Shi, Y.L. Guo, M. Li, X.B. Zhou, Q. Huang, S.Y. Du, Scr. Mater. 141, 99 (2017) 